ARTICLE

https://doi.org/10.1038/s41467-019-11454-9

\title{
Allele specific repair of splicing mutations in cystic fibrosis through AsCas12a genome editing
}

\author{
Giulia Maule (1) 1, Antonio Casini (10 1, Claudia Montagna1, Anabela S. Ramalho², Kris De Boeck ${ }^{2,3}$, \\ Zeger Debyser ${ }^{4}$, Marianne S. Carlon (10) ${ }^{4,6}$, Gianluca Petris (10 ${ }^{1,5,6}$ \& Anna Cereseto (1) 1,6
}

Cystic fibrosis (CF) is an autosomal recessive disease caused by mutations in the CFTR gene. The 3272-26A>G and 3849+10kbC>T CFTR mutations alter the correct splicing of the CFTR gene, generating new acceptor and donor splice sites respectively. Here we develop a genome editing approach to permanently correct these genetic defects, using a single crRNA and the Acidaminococcus sp. BV3L6, AsCas12a. This genetic repair strategy is highly precise, showing very strong discrimination between the wild-type and mutant sequence and a complete absence of detectable off-targets. The efficacy of this gene correction strategy is verified in intestinal organoids and airway epithelial cells derived from CF patients carrying the $3272-26 A>G$ or $3849+10 \mathrm{kbC}>\mathrm{T}$ mutations, showing efficient repair and complete functional recovery of the CFTR channel. These results demonstrate that allele-specific genome editing with AsCas12a can correct aberrant CFTR splicing mutations, paving the way for a permanent splicing correction in genetic diseases.

\footnotetext{
${ }^{1}$ Centre for Integrative Biology (CIBIO), University of Trento, Via Sommarive 9, 38123 Trento, Italy. ${ }^{2}$ Department of Development and Regeneration, CF Centre, Woman and Child, KU Leuven, Herestraat 49, Leuven 3000, Belgium. ${ }^{3}$ Pediatric Pulmonology, Department of Pediatrics, University Hospital Leuven, Herestraat 49, Leuven 3000, Belgium. ${ }^{4}$ Laboratory for Molecular Virology and Drug Discovery, Department of Pharmaceutical and Pharmacological Sciences, KU Leuven, Herestraat 49, Leuven 3000, Belgium. ${ }^{5}$ Present address: Medical Research Council Laboratory of Molecular Biology, Cambridge Biomedical Campus, Francis Crick Avenue, Cambridge CB2 OQH, UK. ${ }^{6}$ These authors jointly supervised this work: Marianne S. Carlon, Gianluca Petris, Anna Cereseto. Correspondence and requests for materials should be addressed to M.S.C. (email: marianne.carlon@kuleuven.be) or to G.P. (email: gpetris@mrc-Imb.cam.ac.uk) or to A.C. (email: anna.cereseto@unitn.it)
} 
C ystic fibrosis (CF) is a lethal autosomal recessive inherited disorder with an approximate frequency of 1 in 2500 births. CF is linked to mutations in the cystic fibrosis transmembrane conductance regulator (CFTR) gene ${ }^{1}$, which encodes a chloride/bicarbonate channel expressed in the apical membrane of epithelial cells. The lack of ion conductance across the membrane of these cells leads to impaired ion and liquid homeostasis, generating a multi-organ disorder. The primary cause of mortality in CF patients is bacterial infections of the airways, provoking chronic lung disease and ultimately respiratory failure ${ }^{2}$. Current CF treatments are not curative and limited to the reduction of clinical symptoms including intestinal-airway blockages and chronic bacterial infections. Recent therapeutic advances were obtained in CF treatments through the development of CFTR correctors and potentiators 3,4 , which however target exclusively few types of mutations including the highly recurrent $\Delta \mathrm{F} 508$.

In search for a cure for $\mathrm{CF}$, several gene therapy approaches have been explored ${ }^{5}$, mostly based on CFTR cDNA gene addition through viral or non-viral vectors ${ }^{6,7}$. Despite promising results obtained in the respiratory tract of animal models ${ }^{8-10}$ and advancements in gene therapy clinical trials ${ }^{11-14}$, curative goals were hampered mainly by low expression levels of the delivered CFTR.

The recent advances in genome editing, with the development of precise and efficient CRISPR-nucleases, have highly accelerated the progress of gene correction for genetic diseases, including $\mathrm{CF}^{15}$. Following initial discovery of the Streptococcus pyogenes Cas9 (SpCas9), several additional CRISPR-nucleases have been discovered with different functional, mechanistic and structural features, expanding the genome editing tool-box ${ }^{16}$. Among these, AsCas12a has been widely used for its different PAM requirements and a natural very high specificity ${ }^{17-20}$.

By means of genome editing, in contrast to the classical gene addition strategies, the correction of the mutated CFTR holds the promise to restore physiological levels of CFTR expression and function. In CF cellular models CFTR genetic repair was obtained through strategies exploiting the cellular homology-directed repair (HDR) pathway ${ }^{21,22}$. Nevertheless, the HDR pathway is not highly active in human cells, thus strongly limiting the clinical efficacy of this application ${ }^{23,24}$. Alternatively, genetic modification by non-homologous end joining (NHEJ) is more efficient and does not require the delivery of a donor DNA template. CRISPR-nucleases have been successfully used to induce NHEJ to knockout genes or genomic regulatory elements ${ }^{25,26}$. This includes permanent repair of splicing defects in CFTR minigene models ${ }^{27}$ as an alternative to the transient and inefficient use of oligonucleotides or spliceosome-mediated RNA trans-splicing (SMaRT) strategies ${ }^{28,29}$.

Here we develop a genome editing strategy to repair 3272$26 \mathrm{~A}>\mathrm{G}(\mathrm{c} .3140-26 \mathrm{~A}>\mathrm{G})$ and $3849+10 \mathrm{kbC}>\mathrm{T}(\mathrm{c} .3718-2477 \mathrm{C}>\mathrm{T})$ CFTR mutations. The $3242-26 \mathrm{~A}>\mathrm{G}$ is a point mutation that creates a new acceptor splice site causing the abnormal inclusion of 25 nucleotides within exon $20^{30,31}$. The resulting mRNA contains a frameshift in CFTR, producing a premature termination codon and consequent expression of a truncated nonfunctional CFTR protein. The $3849+10 \mathrm{kbC}>\mathrm{T}$ mutation creates a novel donor splice site inside intron 22 of the CFTR gene, leading to the insertion of the new cryptic exon of 84 nucleotides, which results in an in-frame stop codon and consequent production of a truncated non-functional CFTR protein ${ }^{32,33}$.

In this study, we harness the AsCas12a nuclease with a single CRISPR RNA (crRNA) to repair the CFTR 3272-26A $>\mathrm{G}$ and $3849+10 \mathrm{kbC}>\mathrm{T}$ splicing defects in different cell types including primary CF patients' airway epithelial cells and intestinal organoids. The genome editing strategy that we develop is highly specific, as demonstrated by a preserved second allele and complete absence of off-target cleavages. CFTR functional recovery by the AsCas12a single crRNA strategy is validated in intestinal organoids derived from CF patients carrying the 3272$26 \mathrm{~A}>\mathrm{G}$ or the $3849+10 \mathrm{kbC}>\mathrm{T}$ mutations, thus highlighting the power of this approach for the permanent correction of genetic diseases caused by deep intronic splicing mutations.

\section{Results}

Splicing correction of a 3272-26A $>$ G minigene model. Minigenes are modeled exon-intron genetic constructs that are useful for studying RNA splicing regulation ${ }^{34-36}$. These constructs are commonly used to study specific cis-acting elements and their binding factors involved in constitutive or alternative splicing regulation ${ }^{37}$.

We generated minigene models to mimic the splicing pattern of the CFTR gene corresponding to the region encompassing part of exon 18, full length exons 19 and 20, and intron 19 (legacy name: exon 16,17a,17b, and intron 17a), either wild-type (pMG3272-26WT) or carrying the 3272-26A $>\mathrm{G}$ mutation (pMG3272-26A $>$ G) (Fig. 1a). The altered or correct splicing pattern produced by the mutated or wild-type minigenes, respectively, was evaluated by RT-PCR and sequencing analyses in transfected HEK293T cells (Supplementary Fig. 1a, b) ${ }^{30}$.

We then designed single guide RNA (sgRNA) ${ }^{16}$ and crRNA, for SpCas9 and AsCas12a, respectively (Supplementary Data 1), to generate single or double cleavages to obtain either isolated indels or deletions within intron 19, near the 3272-26A $>\mathrm{G}$ mutation. The splicing pattern of the pMG3272-26A $>\mathrm{G}$ was evaluated after its transient co-transfection with the sgRNAs/ crRNAs in combination with either SpCas9 or AsCas12a (Supplementary Fig. 2a, b). We observed increased levels of the correct splicing product by using either SpCas9 with at least four sgRNA pairs $(-52 /+9,-47 /-0,-47 /+9,-47 /+10)$ (Supplementary Fig. 2a) or AsCas12a in with crRNAs -2 or +11 used individually or in combination with another crRNA (Supplementary Fig. 2b). Analysis of the deletions induced by sgRNA pairs showed that SpCas9 efficiently cuts the expected DNA fragments (Supplementary Fig. 2c). Conversely, weak deletion products were detected with AsCas12a in samples where splicing was not repaired (compare Supplementary Fig. $2 b$ and $d$ ).

To further validate the activity of SpCas9 or AsCas12a with the selected sgRNAs/crRNAs within a more physiological chromatin context, we tested the splicing correction of CFTR intron 19 in HEK293 cells stably transfected with the pMG3272-26A $>$ G minigene (HEK293/3272-26A $>G$ ). Unexpectedly, all the SpCas9-sgRNA pairs failed to correct the splicing defect, suggesting inefficient cleavage at the chromosomal level (Supplementary Fig. 2e, f). Conversely, the AsCas12a-crRNA +11 generated high amounts of correct transcripts from the pMG3272-26A>G transgene (more than 60\%, Fig. 1b, c and Supplementary Fig. $2 \mathrm{~g}$ ) and efficient DNA editing (67.4\%, Fig. 1d).

The tracking of indels by decomposition (TIDE) analysis of the integrated minigenes ${ }^{38}$, following editing with AsCas12a-crRNA+ 11 , revealed a heterogeneous pool of deletions (Supplementary Fig. 3a). The edited variants were cloned into the pMG3272$26 \mathrm{~A}>\mathrm{G}$ minigene to analyze the individual editing events and their derived splicing products (Fig. 1e). Sequence analysis of the edited sites showed a high frequency of 18 nucleotides deletions, which is consistent with the editing profile of AsCas12a (deletions bigger than four bases ${ }^{20}$ ), along with the persistence of the 3272 $26 \mathrm{~A}>\mathrm{G}$ mutation (Fig. 1e and Supplementary Fig. 3a). Notably, the splicing analysis revealed that the frequent 18 nucleotides deletion (9/34 clones) fully restored the correct splicing (Fig. 1e 
a

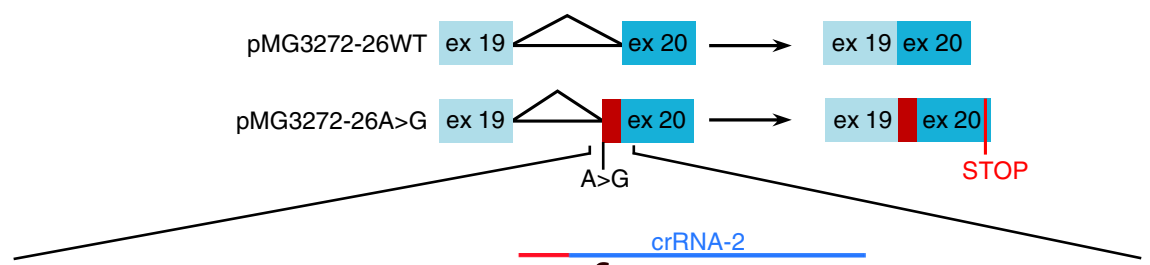

$\widehat{\text { GGTACAAGATATTATGAAATTACATTTTGTGTTTATGTTATTTGCAGTGTTTTCTATGGAAATATTTCACAGGCAGGAGTCCAATTTTC }}$

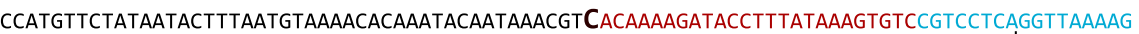
CrRNA +11
ex 20

b
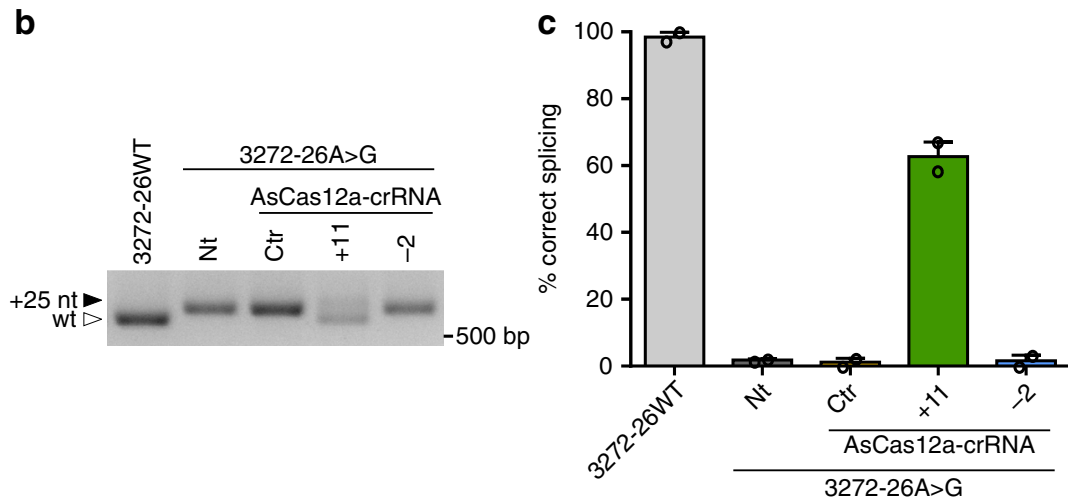

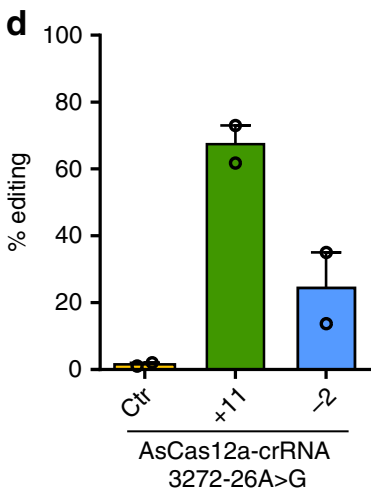

e
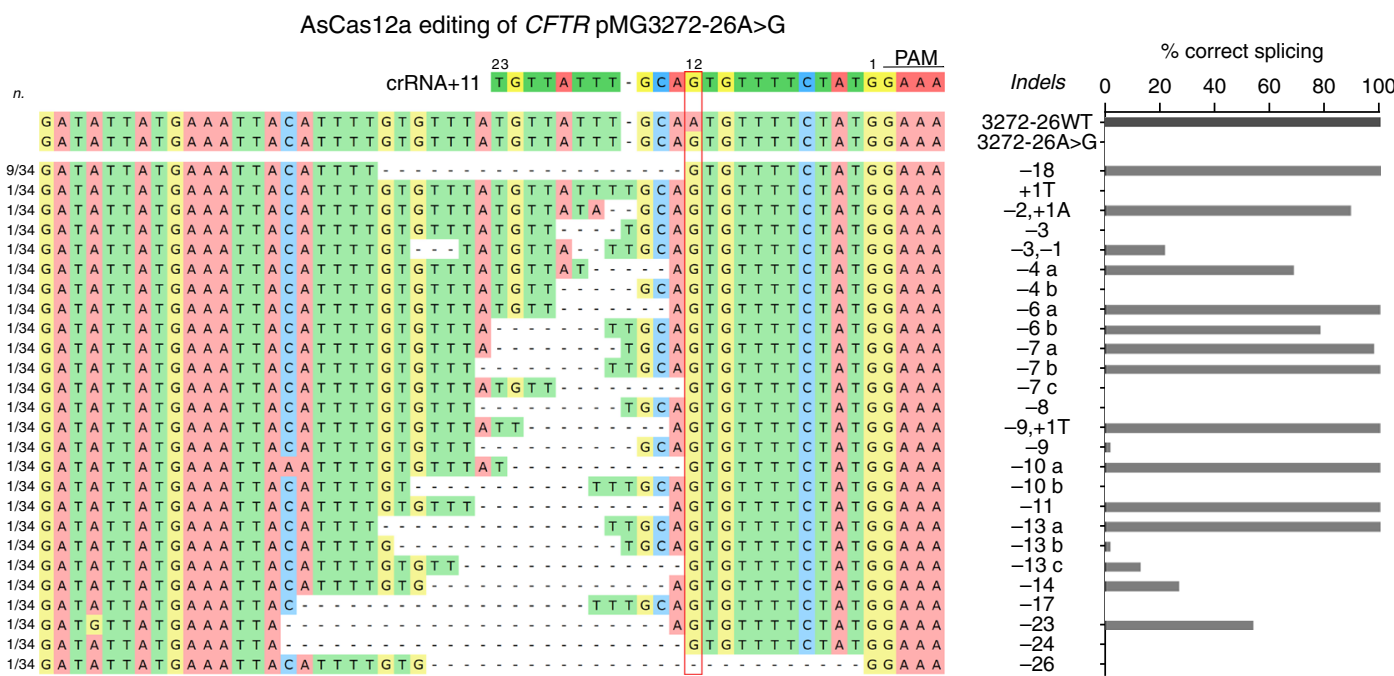

Fig. 1 Splicing correction in CFTR 3272-26A>G minigene model by AsCas12a editing. a Scheme of the CFTR minigenes containing a sequence ( $1.3 \mathrm{~kb}$ ) corresponding to the CFTR region extending from exon 19 to 20 either wild type (pMG3272-26WT) or 3272-26A>G mutated ( $p M G 3272-26 A>G$ ). Exons are shown as boxes, introns as lines; the expected spliced transcripts are represented on the right according to the presence or absence of the 3272$26 \mathrm{~A}>\mathrm{G}$ mutation. The lower panel shows nucleotide sequence and intron-exon boundaries nearby the 3272-26A>G mutation (labeled in bold) and the target AsCas12a-crRNAs position (underlined, with the PAM in red). b Splicing pattern analyzed by RT-PCR in HEK293/pMG3272-26A>G cells following treatments with AsCas12a-crRNA control ( $\mathrm{Ctr}$ ) or specific for the 3272-26A>G mutation ( +11 and -2$)$. Black-solid arrow indicates aberrant splicing, white-empty arrow indicates correct splicing. Representative data of $n=2$ independent experiments. c Percentages of correct splicing measured by densitometry and $\mathbf{d}$ editing efficiency analyzed by TIDE in cells treated as in $\mathbf{b}$. Data are means \pm SEM from $n=2$ independent experiments. e Indels triggered by AsCas12a-crRNA+11. The 3272-26A>G locus from cells edited by crRNA +11 were amplified, cloned in the minigene backbone and Sanger sequenced (34 different clones, left panel), or analyzed as in b for produced splicing pattern (right panel and Supplementary Fig. 3b). pMG3272-26WT and pMG3272-26A>G were used as references

and Supplementary Fig. 3b). Most of the remaining edited sites, occurring at low frequency (1/34 clones), generated a correct splicing, accompanied in few cases by an additional transcript product (Supplementary Fig. 3b). Overall, the large majority $(68 \%)$ of analyzed editing events contributed to the effective restoration of normal splicing in the bulk cell population. In silico analysis 39,40 of the most frequent editing events (above $1 \%$ indel frequency) shows that the large majority of the indels decrease the strength of the cryptic splice sites activated by the 3272-26A>G CF mutation (Supplementary Fig. 3c).

In conclusion, AsCas12a in combination with a single guide RNA (crRNA+11) generates small deletions upstream of the $3272-26 \mathrm{~A}>\mathrm{G}$ mutation in a minigene model, producing efficient recovery of the CF splicing defect. 
a
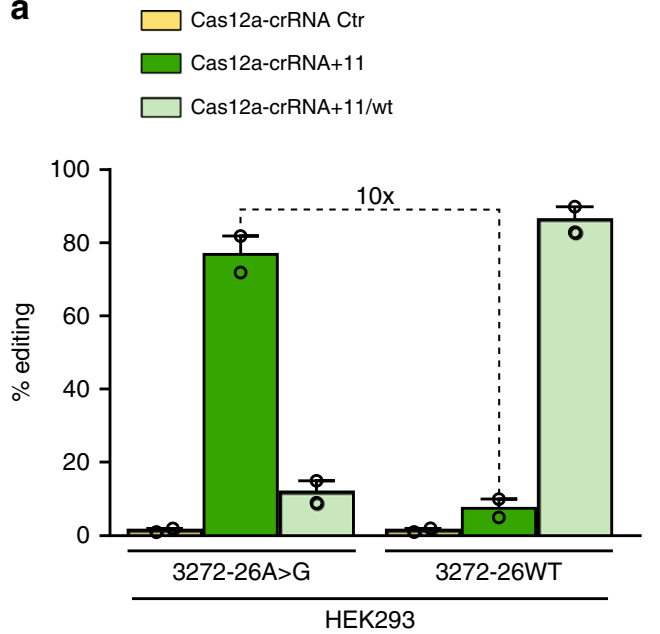

C

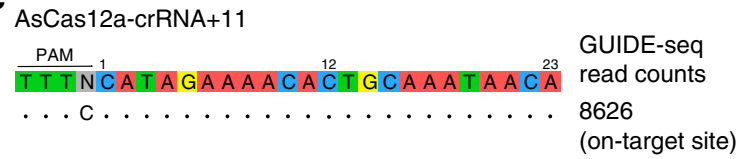

b
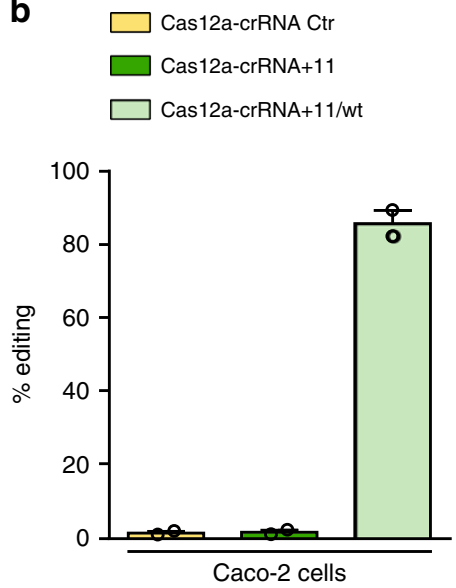

Fig. 2 Target specificity of AsCas12a-crRNA+11. a, b Editing efficiency by TIDE analysis in HEK293/pMG3272-26WT or HEK293/pMG3272-26A>G a and in Caco- 2 cells $\mathbf{b}$ following lentiviral transduction of AsCas12a-crRNA +11 or $+11 / w t$, as indicated. Data are means \pm SEM from $n=2$ independent experiments. c GUIDE-seq analysis of crRNA +11

Precision of the AsCas12a-based 3272-26A $>$ G correction. The large majority of $\mathrm{CF}$ patients are compound heterozygous for the $3272-26 \mathrm{~A}>\mathrm{G}$ mutation, thus requiring a careful evaluation of the potential AsCas12a-crRNA +11 modifications within the other mutant allele, having the wild-type 3272-26 sequence.

The cleavage properties of the AsCas12a-crRNA+11 were analyzed in stable cell lines expressing either pMG3272-26WT or pMG3272-26A >G (HEK293/3272-26WT and HEK293/3272$26 \mathrm{~A}>\mathrm{G}$ cells, respectively). As shown in Fig. 2a, cleavage efficiency of crRNA+11 dropped from $77 \%$, detected in HEK293/3272-26A $>$ G, to $7.5 \%$ in HEK293/3272-26WT, demonstrating at least 10 -fold differential cleavage between the mutant and wild-type allele. Reciprocal experiments with crRNA+11/wt, targeting the CFTR 3272-26WT sequence showed high cleavage efficiency (86.5\%) in HEK293/3272-26WT and low indels formation (12\%) in HEK293/3272-26A $>$ G (Fig. 2a), thus demonstrating high allelic discrimination by AsCas12a with the selected crRNA.

The specificity of the AsCas $12 \mathrm{a}-\mathrm{crRNA}+11$ delivered by lentiviral vectors towards the wild type intron, was further confirmed in Caco-2 epithelial cells endogenously expressing the wild type CFTR gene. Long-term nuclease expression (10 days after transduction), which has been demonstrated to highly favor non-specific cleavages ${ }^{41}$, did not generate any unspecific CFTR editing above TIDE background levels (about $1 \%)^{38}$; whereas AsCas $12 \mathrm{a}$-crRNA $+11 / \mathrm{wt}$ efficiently edited the CFTR gene (86.3\%, Fig. 2b).

To exclude splicing alterations following potential wild-type intronic cleavages, the splicing pattern was evaluated in HEK293/ 3272-26WT and Caco-2 cells: no major alterations were observed following AsCas12a treatment in combination with either crRNA+ 11/wt or crRNA+11 (Supplementary Fig. 4a, b).

The specificity of the AsCas $12 \mathrm{a}$-crRNA +11 was also tested in terms of off-target cleavages by a genome-wide survey, GUIDEseq $^{35,36}$. Off-target profiling of AsCas12a-crRNA +11 genome editing in HEK293/3272-26A>G cells ${ }^{18,42}$ showed very high specificity, as demonstrated by exclusive editing of the $3272-26 \mathrm{~A}>\mathrm{G}$ CFTR locus, while non-specific cleavages in the second allele, or any other genomic loci, could not be detected (Fig. 2c and Supplementary Fig. 4c).

3272-26A $>$ G splicing correction in primary airway cells. The efficacy of the $3272-26 \mathrm{~A}>\mathrm{G}$ correction by AsCas12a-crRNA +11 was further validated in primary airway epithelial cells derived from a patient compound heterozygous for the $3272-26 \mathrm{~A}>\mathrm{G}$ splicing mutation $(3272-26 \mathrm{~A}>\mathrm{G} / \Delta \mathrm{F} 508)$. Human primary airway epithelial cells are a physiologically relevant $2-\mathrm{D}$ model for $\mathrm{CF}$ disease modeling and preclinical testing of CF therapies ${ }^{43,44}$. As expected, two different transcripts were detected in these cells (Fig. 3a), whose difference in size and abundance is consistent with the cells heterozygosity for the 3272-26A $>\mathrm{G}$ mutation and in agreement with previous data ${ }^{30}$. A 13-fold correction of the aberrant 3272-26A $>$ G splicing was obtained by lentiviral delivery of AsCas12a-crRNA+11 either with or without puromycin selection $(+25 \mathrm{nt}$ isoform: $18.8 \%$ control sample, $1.4 \%$ crRNA +11 , $0.3 \%$ crRNA +11 puro; Fig. $3 a$ and Supplementary Fig. $5 \mathrm{a}-\mathrm{c}$ ).

The editing efficiency was evaluated by TIDE analysis revealing $30 \%$ of indels (up to $42 \%$ following puromycin selection, Fig. $3 \mathrm{~b}$ and Supplementary Fig. 5d). Of note, since TIDE sequencing does not distinguish between the two CFTR alleles (3272-26A>G/ $\Delta \mathrm{F} 508)$, the allelic discrimination of our genome editing approach was evaluated by deep sequencing, revealing $78.7 \%$ editing of the $3272-26 \mathrm{~A}>\mathrm{G}$ allele and complete absence of indels in the second CFTR allele (3272-26WT allele, Supplementary Fig. 6a, b). To further evaluate the off-target profile in primary airway epithelial cells, computationally predicted off-target sites with up to four mismatches (12 sites) were analyzed by deep sequencing. Consistent with GUIDE-seq results (Fig. 2c) no off-target cleavages were observed (Supplementary Fig. 6c).

Splicing correction in $3272-26 A>G$ intestinal organoids. Human organoids represent a near-physiological model for translational research ${ }^{45}$. Intestinal organoids from CF patients are 
a

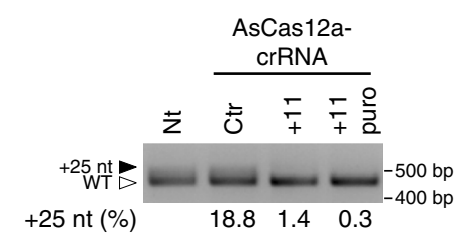

b

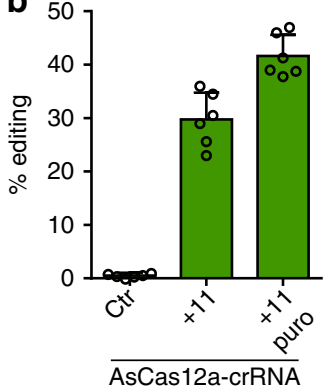

C

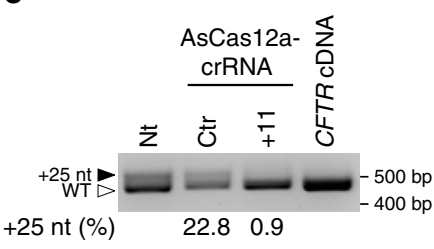

d

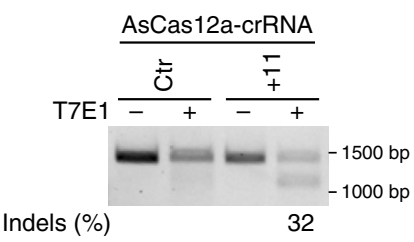

e

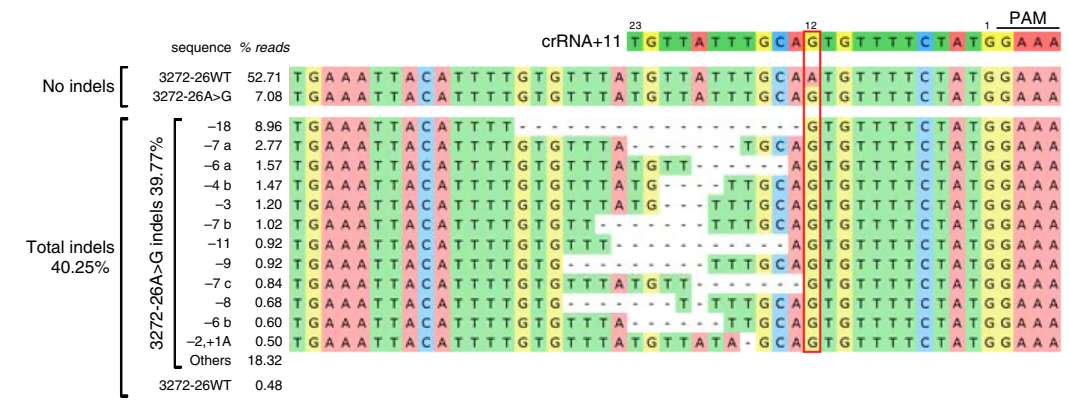

f

3272-26A > G allele $3272-26 W T$ allele

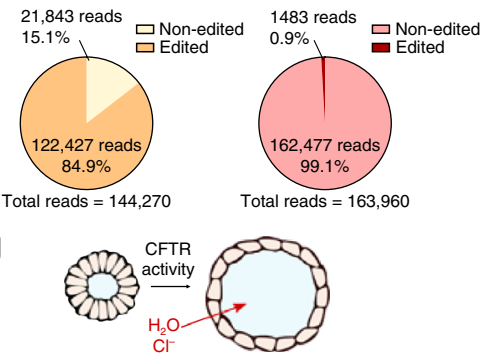

j

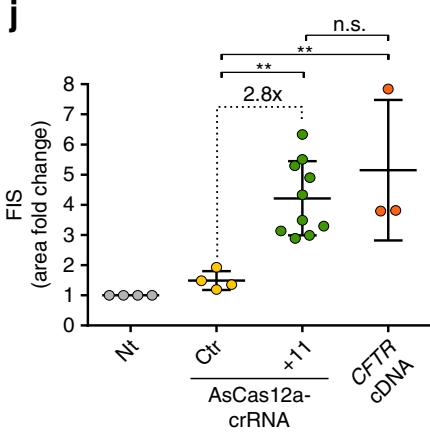

h

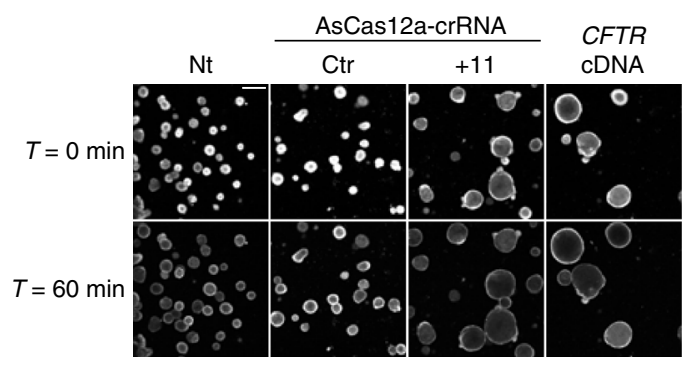

i

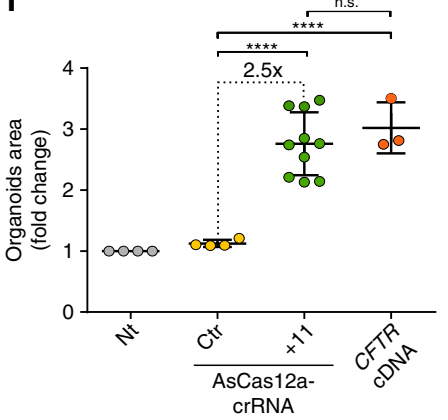

Fig. 3 AsCas12a-crRNA+11 genome editing analysis in 3272-26A>G models. a Splicing pattern analysis by RT-PCR in 3272-26A>G primary airway cells following lentiviral transduction (15 days) of AsCas12a-crRNA control (Ctr) or specific for the 3272-26A>G mutation (+11). Puromycin selection was performed for $72 \mathrm{~h}$ in +11 puro. Black-solid arrow indicates aberrant splicing, white-empty arrow indicates correct splicing. The percentages of aberrant splicing ( 25 nt insertion into mRNA) was measured by chromatogram decomposition analysis (Supplementary Fig. 5). b Percentages of indels generated in intron 20 by crRNA +11 in cells treated as in a measured by TIDE analysis. Data are from $n=2$ independent experiments. c Splicing pattern analysis by RT$P C R$ in 3272-26A>G intestinal organoids following lentiviral transduction (14 days) of AsCas12a-crRNA control (Ctr) or specific for the 3272-26A>G mutation (+11) or with the CFTR cDNA. Black-solid arrow indicates aberrant splicing, white-empty arrow indicates correct splicing. The percentages of aberrant splicing were measured as in a (Supplementary Fig. 6a, b). d Editing efficiency in 3272-26A>G organoids measured by T7E1 assay following lentiviral transduction as in c. e Deep sequencing analysis of the CFTR on-target locus after AsCas12a-crRNA+11 transduction of the 3272-26A>G organoids (average from $n=2$ independent experiments) (Supplementary Data 2). $\mathbf{f}$ Percentage of deep sequencing reads of the edited and non-edited 3272-26A $>$ G or WT alleles from e. $\mathbf{g}$ Schematic representation of CFTR dependent swelling in primary intestinal organoids. $\mathbf{h}$ Representative confocal images of calcein green labeled 3272-26A>G organoids before ( $T=0 \mathrm{~min}$ ) and after ( $T=60 \mathrm{~min}$ ) forskolin-induced swelling (FIS) assay ( $5 \mu M$ forskolin). Scale bar $=200 \mu \mathrm{m}$. i Quantification of organoid area following lentiviral transduction of AsCas12a-crRNA Ctr, AsCas12a-crRNA+11 or with CFTR cDNA as indicated. Each dot represents the average organoid area analyzed for a single well (number of organoids per well: 25-300) from $n=4$ independent experiments. $\mathbf{j}$ Fold change in organoid area before $(T=0 \mathrm{~min})$ and after $(T=60 \mathrm{~min})$ the forskolin-induced swelling (FIS) assay, each dot represents the average increase in organoid area analyzed per well (number of organoids per well: $25-300$ ) from $n=4$ independent experiments. Data are means \pm SD. Statistical analysis was performed using one-way ANOVA; ${ }^{\star \star} P<0.01,{ }^{\star \star \star \star} P<0.0001$, n.s. non-significant

valuable tools to evaluate CFTR channel activity and functional recovery ${ }^{46-48}$.

Encouraged by the splicing correction and allele specificity obtained in minigene cell models and in primary airway epithelial cells, we next evaluated the rescue potential of the CF phenotype by AsCas12a-crRNA+11 in human intestinal organoids compound heterozygous for the 3272-26A $>\mathrm{G}$ mutation (3272$26 \mathrm{~A}>\mathrm{G} / 4218 \mathrm{ins} \mathrm{T})$.

As observed in primary epithelial airway cells, the splicing pattern of CFTR intron 19 in the crRNA control and untreated organoids showed two transcript variants (Fig. 3c). Lentiviral delivery of AsCas12a-crRNA +11 showed nearly complete disappearance of the altered splicing product generated by the $3272-26 \mathrm{~A}>\mathrm{G}$ allele $(+25 \mathrm{nt})$, indicating efficient correction of the aberrant intron 19 splicing (Fig. $3 \mathrm{c}$ and Supplementary Fig. 7a, b). The amount of indels induced by AsCas12a-crRNA +11 was initially evaluated by the T7 Endonuclease I assay, resulting in approximately $30 \%$ editing of the CFTR locus (Fig. 3d), which is consistent with the degree of restored splicing observed in Fig. $3 c$. 
Deep sequencing analysis revealed $40.25 \%$ indels in the CFTR locus $(39.77 \%$ within the $3272-26 \mathrm{~A}>\mathrm{G}$ allele and $0.48 \%$ within the other allele, Fig. 3e), thus confirming the high efficiency of AsCas12a-crRNA +11 editing observed with the T7 Endonuclease I assay (Fig. 3d). Further sequence analysis revealed that $84.9 \%$ of the sequencing reads including the $3272-26 \mathrm{~A}>\mathrm{G}$ mutation contained variable length deletions, while sequencing reads corresponding to the other allele (3272-26WT) contained only $0.9 \%$ indels, thus indicating a 94-fold allelic discrimination (Fig. 3f).

In agreement with previous reports 49,50 , and despite the heterogeneity of the observed editing, the repair events in patient's organoids were largely similar to those observed in pMG3272-26A>G model, with the 18 nucleotide deletion as the most frequent repair (compare Fig. 3e with Fig. 1e). Notably, this 18 nucleotides deletion, as well as most of the other reported indels (with a frequency above $0.5 \%$ of total DNA repair events, Fig. 3e), generated splicing correction when cloned in the pMG3272-26 model (Fig. 1e).

Lumen formation and increased organoid size of intestinal organoids (swelling) depends on the activity of the CFTR anion channel $^{46}$ (schematized in Fig. $3 \mathrm{~g}$ ) and thus can be used to measure the restoration of CFTR function after AsCas12a-crRNA+11 genome editing. Fourteen days post AsCas12a-crRNA+11 treatment the patient's organoids showed a 2.5-fold increased organoid area at steady-state compared to the organoids of control and untreated samples, thus indicating restored channel function following repair of the CFTR 3272-26A $>$ G allele (Fig. 3h, i). Noteworthy, there was no significant difference in organoid area between treatment with AsCas12a-crRNA +11 or transduction of WT CFTR CDNA (Fig. 3i), further supporting the remarkable efficiency of the AsCas12a-crRNA +11 genetic editing in $3272-26 \mathrm{~A}>\mathrm{G}$ phenotypic reversion.

In addition to demonstrating a rescue in steady-state CFTR function (organoid swelling after treatment with AsCas12acrRNA+11), CFTR function was also assessed by the wellestablished forskolin-induced swelling (FIS) assay ${ }^{46}$ (Fig. 3h, j). Consistent with the data in Fig. 3i, the FIS assay revealed an increase in AsCas12a-edited organoid area of 2.8-fold, which is similar to the results obtained with lentiviral delivery of WT CFTR cDNA (Fig. 3j and Supplementary Fig. 7c).

In light of these results, we conclude that AsCas12a-crRNA+11 modifications of the $3272-26 \mathrm{~A}>\mathrm{G}$ defect in patient's organoids allows the repair of the intron 19 splicing defect, leading to full recovery of the endogenous CFTR protein function.

Genetic correction of the CFTR $3849+10 \mathrm{kbC}>\mathrm{T}$ splicing defect. To further evaluate the broader application of the developed AsCas12a-crRNA editing strategy, the CFTR 3849 $+10 \mathrm{kbC}>\mathrm{T}$ splicing mutation was investigated. This genetic variant generates the inclusion of a cryptic exon of 84 nucleotides in the CFTR mature mRNA, which is translated into a truncated defective anion channel ${ }^{33}$.

We generated minigene models (pMG3849+10kbWT and pMG3849+10kbC>T) containing exon 22, part of intron 22 and exon 23 (legacy name: exon 19, intron 19 and exon 20) (schematized in Fig. 4a) that were demonstrated to mimic either wild-type or defective CFTR splicing (Supplementary Fig. 8a-c). The crRNA +14 , targeting the $3849+10 \mathrm{kbC}>\mathrm{T}$ mutation, showed a complete correction of the altered splicing in combination with AsCas12a in the minigene model (Fig. 4b). Moreover, lentiviral transduction of AsCas12a-crRNA +14 in Caco- 2 cells, generated indels (3.5\%) near background levels in the wild-type CFTR gene, while the AsCas12a-crRNA+14/wt, targeting the wild-type sequence in the same region, produced $64 \%$ CFTR editing, thus indicating specificity of the AsCas12a-crRNA+14 towards the mutant allele (Fig. 4c).

To further verify the AsCas12a-crRNA +14 specificity, also in terms of genome-wide off-target activity, GUIDE-seq analysis was performed in HEK293T cells, showing the complete absence of sequence reads in the CFTR locus or in any other off-target site; the 631 sequencing reads corresponding to spontaneous DNA breaks are indicative of the proper execution of the GUIDE-seq assay (Supplementary Fig. 4c).

The CFTR splicing pattern was then analyzed in airway epithelial cells derived from a compound heterozygous patient carrying both the $3849+10 \mathrm{kbC}>\mathrm{T}$ and the $\Delta \mathrm{F} 508$ mutations. The aberrant splicing generated by the $3849+10 \mathrm{kbC}>\mathrm{T}$ mutation $(+84 \mathrm{nt}$ transcript) could be reversed following lentiviral delivery of the mutation specific AsCas12a-crRNA+14 (Fig. 4d), which correlated with $20 \%$ indels (30\% after puromycin selection, Fig.4e). Allelic discrimination of crRNA +14 was evaluated by deep sequencing, revealing $70.7 \%$ cleavages in the $3849+10 \mathrm{kbC}>\mathrm{T}$ locus and fully preserved second CFTR allele $(3849+10 \mathrm{kbWT}$ allele, Supplementary Fig. 9a, b). The editing precision was further evaluated through deep-sequencing analysis of three predicted off-target sites (up to four mismatches), showing complete absence of non-specific cleavages (Supplementary Fig. 9c) as observed with the GUIDE-seq analysis in HEK293Tcells (Supplementary Fig. 4c).

The genome editing strategy was verified in intestinal organoids, derived from a compound heterozygous patient carrying the $3849+10 \mathrm{kbC}>\mathrm{T}$ mutation $(3849+10 \mathrm{kbC}>\mathrm{T} /$ $\triangle \mathrm{F} 508)$. Lentiviral delivery of AsCas12a crRNA +14 produced $30 \%$ of indels in the CFTR loci modifying the aberrant splicing site (Fig. 4f, g and Supplementary Fig. 10a, b), leading to the rescue of organoid swelling as strong as the one observed after CFTR cDNA addition (Fig. 4h, i). Sequencing analysis aimed at evaluating allelic discrimination, confirmed the extreme precision of this genome editing approach (Fig. $4 \mathrm{~g}$ and Supplementary Fig. 9d).

Finally, we evaluated the editing efficacy of SpCas9 using the pMG3849+10kbC>T minigene. We found that, consistently with the most commonly used genome editing strategies ${ }^{27,51}$, SpCas 9 reversed the splicing defects exclusively with sgRNA pairs which deleted the intronic region containing the mutation (Supplementary Fig. 11a, b); in contrast to AsCas12a, SpCas9 combined with an individual sgRNA targeting the mutation $(+1,+5,+18)$ did not repair the splicing defect (Supplementary Fig. 11a).

The best identified sgRNAs pair, $-95 /+119$, was selected among those generating the expected deletion in the CFTR locus and specifically repairing the aberrant splicing (Supplementary Fig. 11c-e). This SpCas9-sgRNA pair induced an increase in organoid area which was significantly lower than the increase observed in organoids after lentiviral delivery of the CFTR cDNA (Supplementary Fig. 11f, g), thus suggesting a lower efficacy than the one obtained with AsCas12a (Fig. 4h, i). Moreover, in contrast to the allele specificity of the single AsCas12-crRNA +14 , the SpCas9-sgRNA pairs, which are necessary for functional correction, produced deletions also in the non-targeted second allele $(\Delta \mathrm{F} 508)$. Therefore, the final genome editing efficacy should be considered diluted over the two alleles. In addition, although our sgRNA pool was designed in silico to minimize the probability of SpCas9 off-target activity ${ }^{52}$, the GUIDE-seq assay for sgRNA +119 revealed 11 off-target sites throughout the genome (Supplementary Figs. 11h and $4 \mathrm{~d}$ ).

In conclusion, similarly to the splicing repair of the 3272 $26 \mathrm{~A}>\mathrm{G}$ variant, the correction of the CFTR $3849+10 \mathrm{kbC}>\mathrm{T}$ splicing defect was efficiently and precisely obtained by using AsCas12a combined with a single allele specific crRNA in CF patient-derived organoids. This strategy was proven superior to 
a

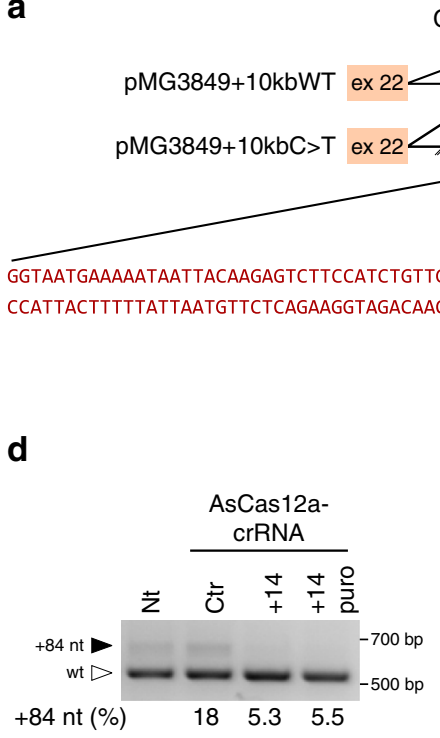

CFTR intron 22 minigene models

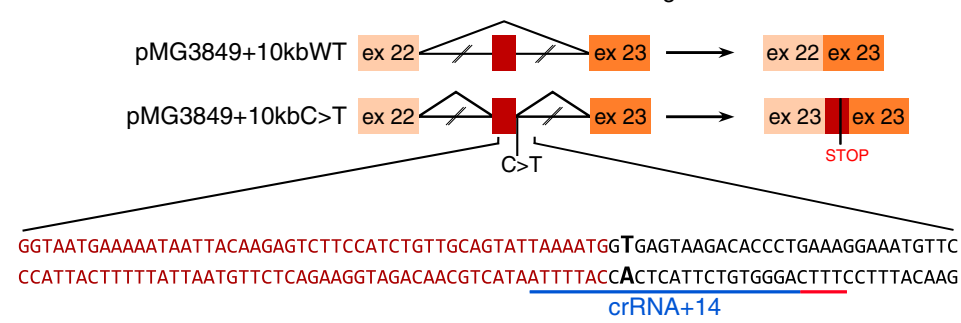

b
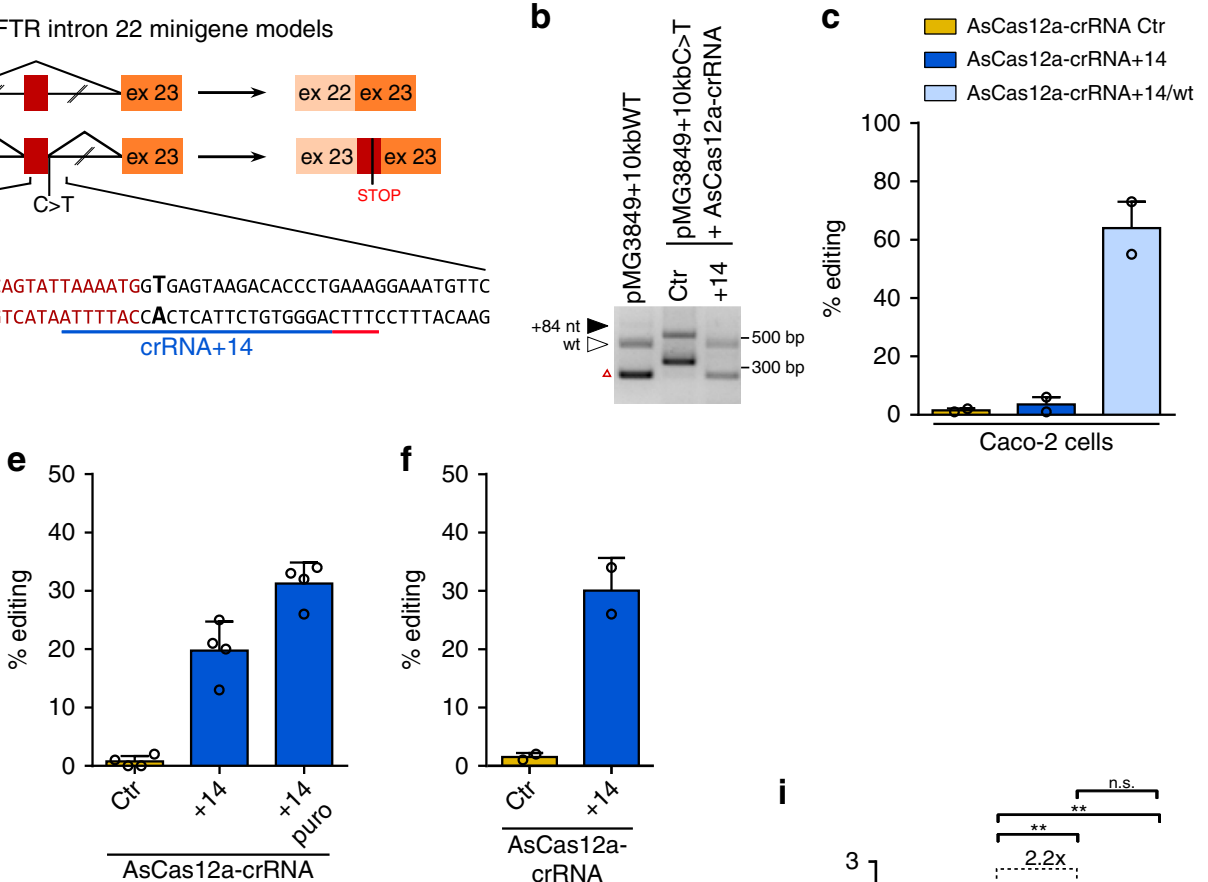

h
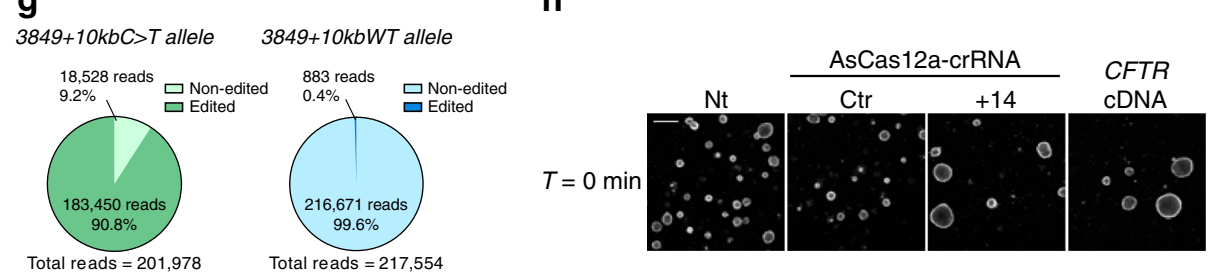

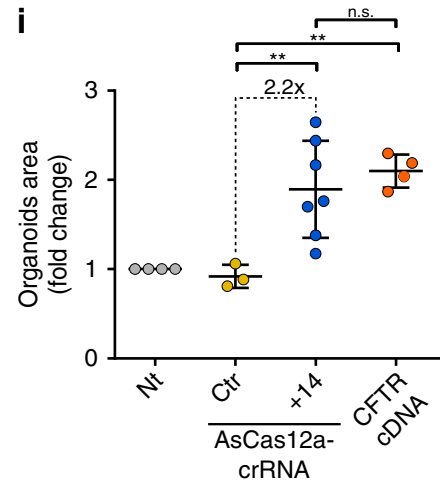

Fig. 4 Correction of 3849+10kbC>T splicing defect by AsCas12a-crRNA+14 editing. a Scheme of CFTR wild type (pMG3849+10kbWT) and 3849+10KbC>T (pMG3849+10kbC>T) minigenes carrying exon 22, portions of intron 22 encompassing the $3849+10 K b C>T$, and exon 23 of the CFTR gene. Exons are shown as boxes and introns as lines; the expected spliced transcripts are represented on the right according to the presence or absence of the 3849+10kbC>T mutation. The lower panel shows nucleotide sequence nearby 3849+10kbC>T mutation (labeled in bold) and the AsCas12a-crRNA+14 target position (underlined, with the PAM in red). b Splicing pattern analyzed by RT-PCR in HEK293/pMG3849+10kbC>T cells following treatments with AsCas12a-crRNA control (Ctr) or specific for the 3272-26A>G mutation (+14). Black-solid arrow indicates aberrant splicing, white-empty arrow indicates correct splicing and red triangle indicates a minigene splicing artifact. c Caco-2 cells lentivirally transduced with AsCas12a-crRNA+14 or +14/wt were analyzed for editing in CFTR intron 22 by SYNTHEGO ICE analysis. Data are means \pm SEM from $n=2$ independent experiments. d Splicing pattern analyzed by RT-PCR in 3849+10kbC $>T$ primary airway cells following lentiviral transduction (15 days) of AsCas12a-crRNA control (Ctr) or specific for the 3849+10kbC $>$ T mutation ( +14$)$. Puromycin selection was performed for $72 \mathrm{~h}$ in +14 puro. The percentage of aberrant splicing ( $84 \mathrm{nt}$ insertion into mRNA) was measured by densitometric analysis. e Percentages of indels in $3849+10 \mathrm{kbC}>T$ primary airway cells measured by TIDE analysis following lentiviral transduction as in $\mathbf{d}$. Data are from $n=2$ independent experiments. $\mathbf{3} 3849+10 \mathrm{~Kb}$ C $>$ T patient derived intestinal organoids were lentivirally transduced with AsCas12a-crRNA control (Ctr) or crRNA +14 and analyzed for intron 22 editing by SYNTHEGO ICE analysis. Data are from $n=1$ experiment. $\mathbf{g}$ Percentage of deep sequencing reads of the edited and non-edited $3849+10 \mathrm{kbC}>\mathrm{T}$ or WT alleles from $\mathbf{f}$. $\mathbf{h}$ Confocal images of calcein green labeled 3849+10KbC>T organoids transduced with AsCas12a-crRNA+14 or CFTR cDNA. Scale bar $200 \mu \mathrm{m}$. i Quantification of organoid area; each dot represents the average area of organoids analyzed in each well (number of organoids per well: $3-30$ ) from $n=1$ experiment. Data are means \pm SD. Statistical analysis was performed using one-way ANOVA; ${ }^{\star \star} P<0.01$, n.s. non-significant

the conventional SpCas9-induced genetic deletion obtained using sgRNA pairs.

\section{Discussion}

The most advanced strategies so far developed for CF gene therapy are based on the delivery, preferably in the lungs, of a copy of the CFTR cDNA to compensate patients' defective CFTR gene. The main limitation of this gene therapy strategy is the low and non-permanent CFTR expression obtained in the affected tissues $^{7,53}$. CFTR expression below therapeutic benefit is mainly due to loss of the trans-gene during the rapid turnover of pulmonary epithelial cells and inefficient lung transduction; conditions that are further worsened by disease symptoms in CF patients. Viral and non-viral vectors have proven valid to deliver the CFTR cDNA in cell and animal models of $\mathrm{CF}^{8,10}$. Among these are the adenoviral and adeno-associated-viral (AAV) delivery systems which, however, are either associated with immune responses or necessitate multiple treatments due to their transient cellular persistence ${ }^{54,55}$. Lentiviral vectors recently reached the clinic for the treatment of different types of genetic diseases. ${ }^{56,57}$. This delivery system with improved genome safety features over the original retroviral vectors, offer significant 
advantages in terms of deliverability in both dividing and nondividing cells. In the CF clinical field recent pre-clinical studies provided encouraging results on lentiviral-mediated CFTR delivery which are leading to the preparation of a first-in-man lentivirus trial in patients with $\mathrm{CF}^{11,58}$.

Latest advances in genome editing, mainly represented by CRISPR-nucleases, offer the unprecedented opportunity to efficiently repair genetic defects within the endogenous CFTR locus to permanently restore the physiological expression of the gene. Furthermore, genetic correction does not require continuous expression of the nuclease thereby bypassing the need for a stable transgene expression ${ }^{59}$. Several methods have been described to limit continuous expression of lentiviral transgenes as natural promoter silencing and self-limiting CRISPR-Cas genetic circuits $41,60,61$.

The CRISPR-nuclease SpCas9 was harnessed to correct the $\Delta$ F508 mutation by homology-directed repair (HDR) ${ }^{22}$. Nevertheless, the low efficiency of HDR in human cells and the requirement of the additional delivery of a DNA donor template pose several hurdles for a future clinical translation. However, the delivery of CRISPR-SpCas9 with multiple sgRNAs was shown to correct CFTR intronic splicing mutation in cellular minigene models by inducing deletions, although quite large in size (around $150-200 \mathrm{bp}$ ), of the intronic mutation and surrounding sequences $^{27}$.

In our study, we developed a genome editing strategy using one of the most precise programmable nucleases ${ }^{18}$, AsCas $12 \mathrm{a}$, that permanently corrects CFTR splicing defects of at least two relevant splicing mutations $(3272-26 \mathrm{~A}>\mathrm{G}$ and $3849+10 \mathrm{kbC}>\mathrm{T})$ in combination with a single crRNA. This approach is based on small deletions (about 4-26 nt) within intronic sequences which remove essential splicing regulatory elements forming aberrant $3^{\prime}$ or $5^{\prime}$ cryptic splice sites. Indeed, our sequencing data indicate that the AsCas12a strategy likely inactivates the cryptic $3^{\prime}$ splice site (poly-pyrimidine-T-rich-tract and last essential $3^{\prime}$-intronic nucleotides) activated by the CFTR 3272-26A>G mutation and the $5^{\prime}$ cryptic donor splice site in intron 22 activated by the 3849 $+10 \mathrm{kbC}>\mathrm{T}$. This was confirmed by the in silico analysis of the splicing signals.

As opposed to AsCas12a, SpCas9 splicing repair required multiple sgRNAs thus strictly depending on efficient concomitant cleavages of the target sites and efficient co-delivery by the delivery vector.

The high-fidelity AsCas12a genetic repair ${ }^{18}$ applied in this study to CFTR splicing defects, resulted in an allele specific and genome-wide off-target free editing. Allelic discrimination was obtained by designing the crRNA target sequence on the mutations $(3272-26 \mathrm{~A}>\mathrm{G}$ and $3849+10 \mathrm{kbC}>\mathrm{T})$, thus providing the relevant advantage of minimizing the potential risk of on-target chromosomal translocations ${ }^{62}$.

We demonstrated that our AsCas12a-based gene correction strategy efficiently corrects the splicing pattern in human primary airway epithelial cells and rescued endogenous CFTR function in patient derived intestinal organoids, which are recognized as a highly valuable preclinical model to predict ex vivo therapeutic efficacy in $\mathrm{CF}$ patients ${ }^{47}$, providing an important milestone for future clinical trials. Even though CFTR modulators (i.e. potentiator VX-770, Kalydeco, Vertex) are used in clinic also for CF patients carrying the splicing mutations of this study, these drugs are associated with side effects and strongly depend on the residual correct splicing of mutant CFTR, which is extremely reduced and variable among patients ${ }^{30-33}$. This explains the urgent need for a permanent correction of physiologic levels of CFTR, potentially reachable with the genome editing approach described in our study.
In conclusion, this work sets a robust proof-of-concept to treat two different deep intronic mutations by genome editing using the AsCas12a-crRNA nuclease system, which can be broadened to other splicing defects in CF and even to other genetic diseases caused by splicing alterations.

\section{Methods}

Plasmids. Wild type and mutated minigenes for $3272-26 \mathrm{~A}>\mathrm{G}$ and $3849+10 \mathrm{kbC}>\mathrm{T}$ mutations were cloned into previously published pcDNA3 and pCI plasmid, respectively 63,64 . Wild type minigenes (pMG3272-26WT and pMG3849 $+10 \mathrm{kbWT}$ ) were obtained by PCR amplification and cloning of target regions from CFTR gene of HEK293T cell. Primers used are listed in Supplementary Data 4. The pMG3272-26WT plasmid includes the last 23 bases of exon 18, full length exons 19 and 20, and intron 19. The addition of the last part of exon 18 was performed by semi-nested PCR: the first PCR was done with oligo TM exon 18 exon 19 hCFTR fw and oligo exon 20 hCFTR rev; the second PCR was done with the forward primer oligo KpnI-AgeI exon 18-19 hCFTR fw and the same reverse oligo. The pMG3849+10kbWT plasmid contains full length exons 22 and 23, and portions of intron 22 as previously described ${ }^{35}$. Mutated minigenes (pMG3272-26A $>\mathrm{G}$ and pMG3849+10kbC>T) were obtained by site-directed mutagenesis of pMG327226WT and pMG3849+10kbWT, for both mutations. Plasmid sequences are described in Supplementary Data 5. Guide RNAs were cloned into pY108 lentiAsCas12a (Addgene Plasmid 84739) or lentiCRISPR v1 (Addgene Plasmid 49535) using BsmBI restriction sites as previously described ${ }^{65}$. These plasmids allow simultaneous delivery of the RNA-guided nuclease and the sgRNA to target cells and contain puromycin as selection marker.

Cell lines. Human colorectal adenocarcinoma cells (Caco-2), HEK293T cells and HEK293 cells stably expressing pMG3272-26WT (HEK293/pMG3272-26WT) or 3272-26A>G cells (HEK293/pMG3272-26A>G) were cultured in Dulbecco's modified Eagle's medium (DMEM; Life Technologies) supplemented with $10 \%$ fetal bovine serum (FBS; Life Technologies), $10 \mathrm{U} / \mathrm{ml}$ antibiotics (PenStrep, Life Technologies) and $2 \mathrm{mM}$ L-glutamine at $37^{\circ} \mathrm{C}$ in a $5 \% \mathrm{CO}_{2}$ humidified atmosphere Puromycin selection was performed using $10 \mu \mathrm{g} / \mathrm{ml}$ for Caco- 2 cells, and $2 \mu \mathrm{g} / \mathrm{ml}$ for HEK293T or HEK293 cells. HEK293T, HEK293, and Caco-2 cells were obtained from American Type Culture Collection (ATCC; www.atcc.org). Stable minigene cell lines (HEK293/3272-26A >G and HEK293/3272-26WT) were produced by transfection of Bgl-II linearized minigene plasmids (pMG3272-26WT or pMG3272-26A>G) in HEK293 cells. Cells were selected with $500 \mu \mathrm{g} / \mathrm{ml}$ of G418, $48 \mathrm{~h}$ after transfection. Single cell clones were isolated and characterized for the expression of the minigene construct.

Transfection and lentiviral transduction of cell lines. Transfection experiment were performed in HEK293T cells seeded (150,000 cells/well) in a 24-well plate and transfected using polyethylenimine (PEI) with $100 \mathrm{ng}$ of minigene plasmids and $700 \mathrm{ng}$ of plasmid encoding for nuclease and sgRNA/crRNA (pY108 lentiAsCas12a or lentiCRISPR v1). After $16 \mathrm{~h}$ incubation cell medium was changed, and samples were collected at 3 days from transfection.

Lentiviral particles were produced in HEK293T cells at $80 \%$ confluency in $10 \mathrm{~cm}$ plates. $10 \mu \mathrm{g}$ of transfer vector (pY108 lentiAsCas12a or lentiCRISPR v1) plasmid, $3.5 \mu \mathrm{g}$ of VSV-G and $6.5 \mu \mathrm{g}$ of $\Delta 8.91$ packaging plasmid were transfected using PEI. After over-night incubation the medium was replaced with complete DMEM. The viral supernatant was collected after $48 \mathrm{~h}$ and filtered in $0.45 \mu \mathrm{m}$ PES filter. Lentiviral particles were concentrated and purified with $20 \%$ sucrose cushion by ultracentrifugation for $2 \mathrm{~h}$ at $4{ }^{\circ} \mathrm{C}$ and $150,000 \times g$. Pellets were resuspended in appropriate volume of OptiMEM. Aliquots were stored at $-80^{\circ} \mathrm{C}$. Vector titres were measured as reverse transcriptase units (RTU) by SG-PERT method 66 .

For transduction experiments HEK293/pMG3272-26WT or HEK293/ pMG3272-26A $>$ G and Caco- 2 cells were seeded $(300,000$ cells/well) in a 12 -well plate and the day after were transduced with three RTU of lentiviral vectors. $48 \mathrm{~h}$ later, cells were selected with puromycin $(2 \mu \mathrm{g} / \mathrm{ml}$ for HEK293 or $10 \mu \mathrm{g} / \mathrm{ml}$ for Caco- 2 cells) and collected 10 days from transduction.

Transcripts analysis. RNA was extracted using TRIzol ${ }^{\mathrm{TM}}$ Reagent (Invitrogen) and resuspended in DEPC-ddH2O. cDNA was obtained starting from $500 \mathrm{ng}$ of RNA using RevertAid Reverse Transcriptase (Thermo Scientific), according to manufacturer's protocol. Target regions were amplified by PCR with Phusion High Fidelity DNA Polymerase (Thermo Fisher). Oligonucleotides are listed in Supplementary Data 4. Uncropped and unprocessed scans are available in the Source Data files.

Detection of nuclease-induced genomic mutations. Genomic DNA was extracted using QuickExtract DNA extraction solution (Epicentre) and the target locus amplified by PCR using Phusion High Fidelity DNA Polymerase (Thermo Fisher). Oligos are listed in Supplementary Data 4. To evaluate indels resulting from cleavage of one gRNA, purified PCR products were sequenced and analyzed 
using the TIDE or the SYNTHEGO ICE software ${ }^{38,67}$. In some experiments DNA editing was measured also by T7 Endonuclease 1 (T7E1) assay (New England BioLabs) following manufacturer's instructions and as previously described ${ }^{41}$.

\section{Primary airway epithelial cell culture and transduction. Primary airway} (bronchial) epithelial cells were derived from CF patients compound heterozygous for 3272-26A $>\mathrm{G}$ splicing mutation $(3272-26 \mathrm{~A}>\mathrm{G} / \Delta \mathrm{F} 508, n=1)$ and for 3849 $+10 \mathrm{~Kb} \mathrm{C}>\mathrm{T}$ splicing mutation $(3849+10 \mathrm{~Kb} \mathrm{C}>\mathrm{T} / \Delta \mathrm{F} 508, n=1)$ (kindly provided by the Primary Cell Culture Service of the Italian Cystic Fibrosis Research Foundation). The Ethics Committee of the Istituto Giannina Gaslini approved this study and informed consent was obtained from all participating CF subjects. Cells were cultured in LHC9/RPMI 1640 (1:1) without serum ${ }^{68}$ and the day before the transduction, 50,000 cells at passage 3 , were seeded into a 24 -well plate previously treated with collagen. Transduction was performed with two RTU of lentiviral vectors. Puromycin selection, where indicated, was performed with $2 \mu \mathrm{g} / \mathrm{ml}, 48 \mathrm{~h}$ post transduction for $72 \mathrm{~h}$. Cells were collected for analysis after 15 days.

Human intestinal organoids culture and transduction. Human intestinal organoids of CF subjects compound heterozygous for $3272-26 \mathrm{~A}>\mathrm{G}$ splicing mutation (3272-26A $>\mathrm{G} / 4218 \mathrm{ins} \mathrm{T}, n=1, \mathrm{CF}-86)$ and for $3849+10 \mathrm{~Kb} \mathrm{C}>\mathrm{T}$ mutation (3849 $+10 \mathrm{~Kb} C>\mathrm{T} / \Delta \mathrm{F} 508, n=1, \mathrm{CF}-110)$ were derived from fresh rectum suction biopsies and cultured as previously described ${ }^{46}$. The Ethics Committee of the University Hospital Leuven approved this study and informed consent was obtained from all participating CF subjects. Organoids cultures, at passages 10-15, were trypsinized to single cell using trypsin $0.25 \%$ EDTA (Gibco), $30,000-40,000$ single cells were resuspended with $25 \mu$ of lentiviral vector $(0.25-1$ RTU) and incubated for $10 \mathrm{~min}$ at $37^{\circ} \mathrm{C}^{8}$. The same amount of Matrigel (Corning) was added and the mix plated in a 96-well plate. After polymerisation of the Matrigel drops $\left(37^{\circ} \mathrm{C}\right.$ for $\left.7 \mathrm{~min}\right)$, they were covered with $100 \mu \mathrm{l}$ of complete organoid medium ${ }^{46}$ containing $10 \mu \mathrm{M}$ of Rock inhibitor (Y-27632 $2 \mathrm{HCl}$, Sigma Aldrich, ref. Y0503) for the first 3 days to ensure optimal outgrowth of single stem cells ${ }^{48}$. Medium was replaced every 2-3 days until the day of organoid analysis.

Analysis of CFTR activity in intestinal organoids. Fourteen days after viral vector transduction, organoids were incubated for $30 \mathrm{~min}$ with $0.5 \mu \mathrm{M}$ calceingreen (Invitrogen, ref. C3-100MP) and analyzed by confocal live cell microscopy with a $\times 5$ objective (LSM800, Zeiss, with Zen Blue software, version 2.3). Steadystate organoids area was determined by calculating the absolute area ( $x y$ plane, $\mu \mathrm{m}^{2}$ ) of each organoid using ImageJ software through the Analyze Particle algorithm. Defective particles with an area $<1500$ or $3000 \mu \mathrm{m}$ for $3272-26 \mathrm{~A}>\mathrm{G}$ or 3849 $+10 \mathrm{~Kb} C>\mathrm{T}$, respectively, were excluded from the analysis. Data were averaged for each different experiment and plotted in a box plot representing means \pm SD.

The FIS assay was performed by stimulating organoids with $5 \mu \mathrm{M}$ of forskolin and analyzed by confocal live cell microscopy at $37^{\circ} \mathrm{C}$ for $60 \mathrm{~min}$ (one image every $10 \mathrm{~min})$. The organoid area ( $x y$ plane) at different time points was calculated using ImageJ, as described above.

GUIDE-seq. GUIDE-seq experiments were performed as previously described ${ }^{42}$ Briefly, $2 \times 10^{5}$ HEK293T cells were transfected using Lipofectamine 3000 transfection reagent (Invitrogen) with $1 \mu \mathrm{g}$ of lenti Cas12a plasmid (pY108) and $10 \mathrm{pmol}$ of dsODNs ${ }^{42}$. The day after transfection cells were detached and selected with $2 \mu \mathrm{g} /$ $\mathrm{ml}$ puromycin. Four days after transfection cells were collected and genomic DNA extracted using DNeasy Blood and Tissue kit (Qiagen) following manufacturer's instructions. Using Bioruptor Pico sonicatin device (Diagenode) genomic DNA was sheared to an average length of $500 \mathrm{bp}$. Library preparations were performed with the original adapters and primers according to previous work. Libraries were quantified with the Qubit dsDNA High Sensitivity Assay kit (Invitrogen) and sequenced with the MiSeq sequencing system (Illumina) using an Illumina Miseq Reagent kit V2-300 cycles $(2 \times 150$ bp paired-end). Raw sequencing data (FASTQ files) were analyzed using the GUIDE-seq computational pipeline ${ }^{42}$. After demultiplexing, putative PCR duplicates were consolidated into single reads. Consolidated reads were mapped to the human reference genome GrCh37 using BWA-MEM; reads with mapping quality lower than 50 were filtered out. Upon the identification of the genomic regions integrating double-stranded oligodeoxynucleotide (dsODNs) in aligned data, off-target sites were retained if at most seven mismatches against the target were present and if absent in the background controls. Visualization of aligned off-target sites is available as a color-coded sequence grid ${ }^{23,26}$. GUIDE-seq data are listed in Supplementary Data 3.

Targeted deep sequencing. The loci of interest were amplified using Phusion high-fidelity polymerase (Thermo Scientific) from genomic DNA extracted from human intestinal organoids (3272-26A $>\mathrm{G} / 4218$ insT) 14 days after transduction with lentiAsCas12a-crRNA $+11,+14$ or Ctr, either in organoids (on-target) or airway cells (on and off-target). Amplicons were indexed by PCR using Nextera indexes (Illumina), quantified with the Qubit dsDNA High Sensitivity Assay kit (Invitrogen), pooled in near-equimolar concentrations and sequenced on an Illumina Miseq system using an Illumina Miseq Reagent kit V3-150 cycles (150 bp single read). Primers used to generate the amplicons are reported in Supplementary Data 4. Raw sequencing data (FASTQ files) were analyzed using CRISPResso online tool ${ }^{69}$, by setting Windows size $=3$, Minimum average read quality $($ phred33 scale $)=30$ and minimum single bp quality (phred33 scale) $=10$ (Supplementary Data 2).

In silico off-target analysis. Off-target for crRNA +11 and +14 were analyzed by Cas-OFFinder online algorithm, by selecting: AsCpf1 from Acidaminococcus or LbCpf1 from Lachnospiraceae 5'-TTTV-3', mismatch number $\leq 4$, DNA bulge size $=0$ and as a target genome the Homo sapiens (GRCh38/hg38)-Human.

In silico splicing prediction. For wild-type, mutated and edited CFTR gene sequences, a region of 400 bp spanning either the $3272-26 \mathrm{~A}>\mathrm{G}$ or $3849+10 \mathrm{~Kb} \mathrm{C}>\mathrm{T}$ locus was analyzed by HSF and MaxEnt prediction algorithms ${ }^{39,40}$ available at the Human Splicing Finder website (www.umd.be/HSF3/). Splice sites score were normalized to the score of the $3272-26 \mathrm{~A}>\mathrm{G}$, or $3849+10 \mathrm{~Kb} \mathrm{C}>\mathrm{T}$ splice site (Supplementary Data 6).

Statistical analyses. Statistical analyses were performed by GraphPad Prism version 6. For organoids experiments ordinary one-way analysis of variance (ANOVA) was performed. For the in silico prediction analyses Shapiro-Wilk test was used to verify the distribution of the data. Significance of the data was calculated by two-tailed Wilcoxon signed-rank test. Differences were considered statistically different at $P<0.05$.

Reporting summary. Further information on research design is available in the Nature Research Reporting Summary linked to this article.

\section{Data availability}

GUIDE-seq and targeted deep-sequencing data have been deposited at BioProject (https://www.ncbi.nlm.nih.gov/bioproject/) under the accession number PRJNA551109. The source data underlying Figs. 1b-e, 2a, b, 3a-d, j, k, 4b-f, i and Supplementary Figs. 1a, 2e-f, 3a, b, 4a, b, 5a-c, 7b, c, 8a, 11a-e, g are provided as a Source Data file. All other relevant data are available from the authors upon reasonable request.

Received: 23 August 2018 Accepted: 5 July 2019

Published online: 07 August 2019

\section{References}

1. Riordan, J. R. et al. Identification of the cystic fibrosis gene: cloning and characterization of complementary DNA. Science (80-.) 245, 1066-1073 (1989).

2. Cutting, G. R. Cystic fibrosis genetics: from molecular understanding to clinical application. Nat. Rev. Genet. 16, 45-56 (2015).

3. Goor, F. Van et al. Correction of the F508del-CFTR protein processing defect in vitro by the investigational drug VX-809. PNAS 7, 159-163 (2011).

4. Goor, F. Van et al. Rescue of CF airway epithelial cell function in vitro by a CFTR potentiator, VX-770. PNAS 20, 16-17 (2014).

5. Carlon, M. S., Vidović, D. \& Birket, S. Roadmap for an early gene therapy for cystic fibrosis airway disease. Prenat. Diagn. 37, 1181-1190 (2017).

6. Sondhi, D., Stiles, K. M., De, B. P. \& Crystal, R. G. Genetic modification of the lung directed toward treatment of human disease. Hum. Gene Ther. 28, 3-84 (2017).

7. Alton, E. W. F. W. et al. Genetic medicines for CF: Hype versus reality. Pediatr. Pulmonol. 51, S5-S17 (2016).

8. Vidovic, D. et al. rAAV-CFTRDR rescues the cystic fibrosis phenotype in human intestinal organoids and cystic fibrosis mice. Am. J. Respir. Crit. Care Med. 193, 288-298 (2016)

9. McNeer, N. A. et al. Nanoparticles that deliver triplex-forming peptide nucleic acid molecules correct F508del CFTR in airway epithelium. Nat. Commun. 6, 1-11 (2015)

10. Robinson, E. et al. Lipid nanoparticle-delivered chemically modified mRNA restores chloride secretion in cystic fibrosis. Mol. Ther. 26, 2034-2046 (2018)

11. Alton, E. W. F. W. et al. Preparation for a first-in-man lentivirus trial in patients with cystic fibrosis. Thorax 72, 137-147 (2017).

12. Pillay, S. et al. An essential receptor for adeno-associated virus infection. Nature 530, 108-112 (2016).

13. Alton, E. W. et al. Repeated nebulisation of non-viral CFTR gene therapy in patients with cystic fibrosis: a randomised, double-blind, placebo-controlled, phase 2b trial. Lancet Respir. Med. 3, 684-691 (2015).

14. Moss, R. B. et al. Repeated aerosolized AAV-CFTR for treatment of cystic fibrosis: a Randomized Placebo-Controlled Phase 2B trial. Hum. Gene Ther. 18, 726-732 (2007). 
15. Razzouk, S. CRISPR-Cas9: a cornerstone for the evolution of precision medicine. Ann. Hum. Genet. 1-27 (2018). https://doi.org/10.1111/ahg.12271.

16. Knott, G. J. \& Doudna, J. A. CRISPR-Cas guides the future of genetic engineering. Science (80-.) 361, 866-869 (2018).

17. Gao, L. et al. Engineered Cpf1 variants with altered PAM specificities. Nat. Biotechnol. 35, 789-792 (2017).

18. Kleinstiver, B. P. et al. Genome-wide specificities of CRISPR-Cas Cpf1 nucleases in human cells. Nat. Biotechnol. 34, 869-874 (2016).

19. Swarts, D. C. \& Jinek, M. Cas9 versus Cas12a/Cpf1: Structure-function comparisons and implications for genome editing. Wiley Inter. Rev. RNA 9, e1481 (2018)

20. Kim, D. et al. Genome-wide analysis reveals specificities of Cpf1 endonucleases in human cells. Nat. Biotechnol. 34, 863-868 (2016).

21. Bednarski, C. et al. Targeted integration of a super-exon into the CFTR locus leads to functional correction of a cystic fibrosis cell line model. PLoS One 11, 1-15 (2016)

22. Schwank, G. et al. Functional repair of CFTR by CRISPR/Cas9 in intestinal stem cell organoids of cystic fibrosis patients. Cell Stem Cell 13, 653-658 (2013).

23. Montagna, C. et al. VSV-G-enveloped vesicles for traceless delivery of CRISPR-Cas9. Mol. Ther. Nucleic Acids 12, 453-462 (2018).

24. Genovese, P. et al. Targeted genome editing in human repopulating haematopoietic stem cells. Nature 510, 235-240 (2014).

25. Romanel, A. et al. Inherited determinants of early recurrent somatic mutations in prostate cancer. Nat. Commun. 8, 1-9 (2017).

26. Casini, A. et al. A highly specific SpCas9 variant is identified by in vivo screening in yeast. Nat. Biotechnol. 36, 265-271 (2018).

27. Sanz, D. J., Hollywood, J. A., Scallan, M. F. \& Harrison, P. T. Cas9/gRNA targeted excision of cystic fibrosis-causing deep-intronic splicing mutations restores normal splicing of CFTR mRNA. PLoS One 12, 1-13 (2017).

28. Friedman, K. J. et al. Correction of aberrant splicing of the cystic fibrosis transmembrane conductance regulator (CFTR) gene by antisense oligonucleotides. J. Biol. Chem. 274, 36193-36199 (1999).

29. Mansfield, S. G. et al. 5' Exon replacement and repair by spliceosomemediated RNA trans-splicing. RNA 9, 1290-1297 (2003).

30. Beck, S. et al. Cystic fibrosis patients with the $3272-26 \mathrm{~A} \rightarrow \mathrm{G}$ mutation have mild disease, leaky alternative mRNA splicing, and CFTR protein at the cell membrane. Hum. Mutat. 14, 133-144 (1999).

31. Amaral, M. D. et al. Cystic fibrosis patients with the $3272-26 \mathrm{~A}>\mathrm{G}$ splicing mutation have milder disease than F508del homozygotes: a large European study. J. Med. Genet. 38, 777-783 (2001).

32. Highsmith, W. E. 1 et al. A novel mutation in the cystic fibrosis gene in patients with pulmonary disease but normal sweat chloride concentrations. $N$. Engl. J. Med. 331, 974-980 (1994).

33. Duguépéroux, I. \& De Braekeleer, M. The CFTR $3849+10 \mathrm{kbC}->$ T and 2789 $+5 \mathrm{G}->\mathrm{A}$ alleles are associated with a mild CF phenotype. Eur. Respir. J. 25, 468-473 (2005).

34. Buratti, E., Stuani, C., De Prato, G. \& Baralle, F. E. SR protein-mediated inhibition of CFTR exon 9 inclusion: Molecular characterization of the intronic splicing silencer. Nucleic Acids Res. 35, 4359-4368 (2007).

35. Nissim-Rafinia, M., Chiba-Falek, O., Sharon, G., Boss, A. \& Kerem, B. Cellular and viral splicing factors can modify the splicing pattern of CFTR transcripts carrying splicing mutations. Hum. Mol. Genet. 9, 1771-1778 (2000).

36. Kashima, T., Rao, N., David, C. J. \& Manley, J. I. hnRNP A1 functions with specificity in repression of SMN2 exon 7 splicing. Hum. Mol. Genet. 16, 3149-3159 (2007).

37. Cooper, T. A. Use of minigene systems to dissect alternative splicing elements. Methods 37, 331-340 (2005).

38. Brinkman, E. K., Chen, T., Amendola, M. \& Van Steensel, B. Easy quantitative assessment of genome editing by sequence trace decomposition. Nucleic Acids Res. 42, 1-8 (2014).

39. Hamroun, D. \& Lalande, M. Human Splicing Finder: an online bioinformatics tool to predict splicing signals. Nucleic Acids Res. 37, 1-14 (2009).

40. Yeo, G. \& Burge, C. B. Maximum entropy modeling of short sequence motifs with applications to RNA splicing signals. J. Comput. Biol. 11, 377-394 (2004).

41. Petris, G. et al. Hit and go CAS9 delivered through a lentiviral based selflimiting circuit. Nat. Commun. 8, 1-9 (2017).

42. Tsai, S. Q. et al. GUIDE-seq enables genome-wide profiling of off-target cleavage by CRISPR-Cas nucleases. Nat. Biotechnol. 33, 187-198 (2015).

43. Awatade, N. T. et al. Human primary epithelial cell models: promising tools in the era of cystic fibrosis personalized medicine. Front. Pharm. 9, 1429 (2018).

44. Gianotti, A., Delpiano, L. \& Caci, E. In vitro methods for the development and analysis of human primary airway epithelia. Front. Pharm. 9, 1-12 (2018).

45. Fatehullah, A., Tan, S. H. \& Barker, N. Organoids as an in vitro model of human development and disease. Nat. Cell Biol. 18, 246-254 (2016).

46. Dekkers, J. F. et al. A functional CFTR assay using primary cystic fibrosis intestinal organoids. Nat. Med. 19, 939-945 (2013).
47. Dekkers, J. F. et al. Characterizing responses to CFTR-modulating drugs using rectal organoids derived from subjects with cystic fibrosis. Sci. Transl. Med. 8 , $344 \mathrm{ra} 84$ (2016).

48. Sato, T. et al. Long-term expansion of epithelial organoids from human colon, adenoma, adenocarcinoma, and Barrett's epithelium. Gastroenterology 141, 1762-1772 (2011)

49. Shen, M. W. et al. Predictable and precise template-free CRISPR editing of pathogenic variants. Nature 563, 646-651 (2018).

50. van Overbeek, M. et al. DNA repair profiling reveals nonrandom outcomes at Cas9-mediated breaks. Mol. Cell 63, 633-646 (2016).

51. Ruan, G. X. et al. CRISPR/Cas9-mediated genome editing as a therapeutic approach for Leber congenital amaurosis 10. Mol. Ther. 25, 331-341 (2017).

52. Doench, J. G. et al. Optimized sgRNA design to maximize activity and minimize off-target effects of CRISPR-Cas9. Nat. Biotechnol. 34, 184-191 (2016).

53. Sondhi, D., Stiles, K. M., De, B. P. \& Crystal, R. G. Genetic modification of the lung directed toward treatment of human disease. Hum. Gene Ther. 28, 3-84 (2017).

54. Carlon, M. S. et al. Immunological ignorance allows long-term gene expression after perinatal recombinant adeno-associated virus-mediated gene transfer to murine airways. Hum. Gene Ther. 25, 517-528 (2014).

55. Giacca, M. \& Zacchigna, S. Virus-mediated gene delivery for human gene therapy. J. Control. Release 161, 377-388 (2012).

56. Mamcarz, E. et al. Lentiviral gene therapy combined with low-dose busulfan in infants with SCID-X1. N. Engl. J. Med. 380, 1525-1534 (2019).

57. Biffi, A. et al. Lentiviral hematopoietic stem cell gene therapy benefits metachromatic leukodystrophy Science. 2013 Aug 23, 341, https://doi.org/ 10.1126/science.1233158 1233158. Epub 2013 Jul 11.

58. Marquez Loza, L., Yuen, E. \& McCray, P. Lentiviral Vectors for the Treatment and Prevention of Cystic Fibrosis Lung Disease. Genes 10, 218 (2019).

59. Zetsche, B., Heidenreich, M., Mohanraju, P. \& Fedorova, I. Multiplex gene editing by CRISPR-Cpf1 through autonomous processing of a single crRNA array. Nat. Biotechnol. https://doi.org/10.1101/049122 (2016).

60. Herbst, F. et al. Extensive methylation of promoter sequences silences lentiviral transgene expression during stem cell differentiation in vivo. Mol. Ther. 20, 1014-1021 (2012).

61. Gill, D. R. et al. Increased persistence of lung gene expression using plasmids containing the ubiquitin $\mathrm{C}$ or elongation factor la promoter. Gene Ther. 8, 1539-1546 (2001).

62. Frock, R. L. et al. Genome-wide detection of DNA double-stranded breaks induced by engineered nucleases. Nat. Biotechnol. 33, 179-186 (2015).

63. Cesaratto, F., López-Requena, A., Burrone, O. R. \& Petris, G. Engineered tobacco etch virus (TEV) protease active in the secretory pathway of mammalian cells. J. Biotechnol. 212, 159-166 (2015).

64. Alanis, E. F. et al. An exon-specific U1 small nuclear RNA (snRNA) strategy to correct splicing defects. Hum. Mol. Genet. 21, 2389-2398 (2012).

65. Shalem, O. et al. Genome-scale CRISPR-Cas9 knockout screening in human cells. Science (80-.) 343, 84-87 (2014).

66. Casini, A., Olivieri, M., Vecchi, L., Burrone, O. R. \& Cereseto, A. Reduction of HIV-1 infectivity through endoplasmic reticulum-associated degradationmediated Env depletion. J. Virol. 89, 2966-2971 (2015).

67. Hsiau, T. et al. Inference of CRISPR edits from Sanger trace data. bioRxiv https://doi.org/10.1101/251082 (2018).

68. Sondo, E. et al. Pharmacological inhibition of the ubiquitin ligase RNF5 rescues F508del-CFTR in cystic fibrosis airway epithelia. Cell Chem. Biol. 25, 891-905.e8 (2018)

69. Pinello, L. et al. Analyzing CRISPR genome editing experiments with CRISPResso. Nat. Biotechnol. 34, 695-697 (2016).

\section{Acknowledgements}

The authors are grateful to Daniele Arosio for helpful discussion throughout the development of this study and to Liesbeth De Keersmaecker for her excellent technical assistance. We thank Francesca Demichelis and Davide Prandi for continuing support in GUIDE-seq analyses. This work was supported by the Italian Cystic Fibrosis Foundation grant FFC\#1/2017 adopted by Associazione Trentina Fibrosi Cistica in ricordo di Maria Cainelli e Romana Petrolli, delegazioni FCC di Imola e Romagna, di Alborello, Lucca, and by intramural funding from the University of Trento. We are grateful to Primary Cell Culture Serviceof the Italian Cystic Fibrosis Research Foundation at the Laboratory of Medical Genetics, G. Gaslini Institute, Genova, Italy, for CF primary cells. Work at the KU Leuven was supported by grants from the King Baudouin Foundation, Belgium [Fund Alphonse and Jean Forton, 3M140231] and a KU Leuven C3 grant OPIT-CF.

\section{Author contributions}

G.M., G.P., and A.C. conceived the study and designed the experiments; G.M. performed most experiments; M.S.C., A.S.R., K.D.B., and Z.D. contributed to organoid experiments; 
G.M., G.P., A.Ca., C.M., M.S.C., and A.C. analyzed the data; G.M., G.P., M.S.C., Z.D., and A.C. wrote and edited the paper. All authors read, corrected, and approved the final manuscript.

\section{Additional information}

Supplementary Information accompanies this paper at https://doi.org/10.1038/s41467019-11454-9.

Competing interests: G.M., A.Ca., M.S.C., G.P. and A.C. are listed as inventors on a patent application related to this work. A.C. and G.P. are scientific advisor to Alia

Therapeutics. A.Ca. and G.P. are cofounder af Alia Therapeutics. A.Ca. is a current employee of Alia Therapeutics. The remaining authors declare no competing interests.

Reprints and permission information is available online at http://npg.nature.com/ reprintsandpermissions/

Peer review information: Nature Communications thanks the anonymous reviewers for their contribution to the peer review of this work.
Publisher's note: Springer Nature remains neutral with regard to jurisdictional claims in published maps and institutional affiliations.

(c) (i) Open Access This article is licensed under a Creative Commons Attribution 4.0 International License, which permits use, sharing, adaptation, distribution and reproduction in any medium or format, as long as you give appropriate credit to the original author(s) and the source, provide a link to the Creative Commons license, and indicate if changes were made. The images or other third party material in this article are included in the article's Creative Commons license, unless indicated otherwise in a credit line to the material. If material is not included in the article's Creative Commons license and your intended use is not permitted by statutory regulation or exceeds the permitted use, you will need to obtain permission directly from the copyright holder. To view a copy of this license, visit http://creativecommons.org/ licenses/by/4.0/.

(c) The Author(s) 2019 